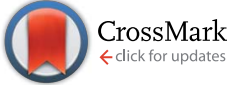

Cite this: Chem. Sci., 2016, 7, 5939

\title{
Facile hydrogen atom transfer to iron(III) imido radical complexes supported by a dianionic pentadentate ligand $\dagger$
}

\author{
Denis M. Spasyuk, ${ }^{\text {a }}$ Stephanie H. Carpenter, ${ }^{\text {b }}$ Christos E. Kefalidis, ${ }^{c}$ Warren E. Piers, ${ }^{\text {*a }}$ \\ Michael L. Neidig*b and Laurent Maron*c
}

Received 31st March 2016

Accepted 28th May 2016

A dianionic tetrapodal pentadentate diborate ligand is introduced. This ligand forms a high spin neutral iron(II) complex that reacts with a variety of organoazides to yield transient Fe(II) imido radicals that are extremely potent hydrogen atom abstractors. The nature of these species is supported by full characterization of the Fe(III) amido products, kinetic studies, density functional computations and Mössbauer spectroscopy on the $-\mathrm{C}_{6} \mathrm{H}_{4}-p-{ }^{t} \mathrm{Bu}$ substituted derivative.

www.rsc.org/chemicalscience

\section{Introduction}

Tetrapodal pentadentate ligands can be used to provide a defined platform for small molecule activation and catalysis. ${ }^{1}$ One successful ligand design is the polypyridyl $\mathrm{PY}_{5}$ system first reported by the Stack $^{2}$ and Feringa ${ }^{3}$ groups. This neutral ligand coordinates to metals from across the periodic table $\mathbf{4}^{4}$ and its oxidative stability allows access to a range of metal oxidation states, ${ }^{5}$ a key feature for applications in catalysis. ${ }^{6}$ This ligand framework has been successfully employed in catalyst systems that generate dihydrogen ${ }^{7-10}$ or oxygen ${ }^{11-13}$ from water, using earth abundant metals such as molybdenum and cobalt. ${ }^{\mathbf{1 4}}$ More recently, Gardinier has introduced a related ligand system, $\mathrm{Pz}_{4} \mathrm{Py}$, incorporating pyrazolyl donors instead of pyridyl moieties. ${ }^{15}$

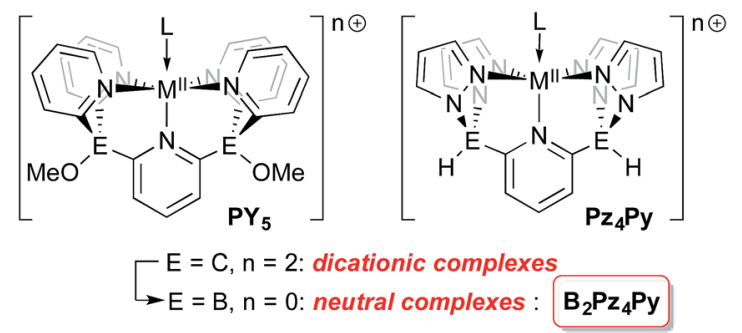

a University of Calgary, Department of Chemistry, 2500 University Drive N.W., Calgary, Alberta, Canada, T2N 1N4. E-mail: wpiers@ucalgary.ca

${ }^{b}$ Department of Chemistry, University of Rochester, Rochester, New York 14627, USA 'LPCNO, Université de Toulouse, INSA, UPS, CNRS, 135 avenue de Rangueil, F-31077 Toulouse, France

$\dagger$ Electronic supplementary information (ESI) available: Synthesis, characterization data, experimental and computational details. Crystallographic data for $\left[\mathbf{B}_{2} \mathbf{P z}_{\mathbf{4}} \mathbf{L i P y H}\right]_{2}, \mathbf{1} \cdot \mathbf{T H F}, \mathbf{1}, \mathbf{2} \cdot \mathbf{A r}, \mathbf{2} \cdot \mathbf{A d}$ and $\mathbf{4}$. CCDC 1439938 and 1439242-1439246. For ESI and crystallographic data in CIF or other electronic format see DOI: 10.1039/c6sc01433j
The overall neutral charge of the $\mathrm{PY}_{5}$ and $\mathrm{Pz}_{4} \mathrm{Py}$ ligands necessarily means that higher oxidation state intermediates bear positive charges and are fleeting, highly reactive species. Rendering ligands anionic ${ }^{\mathbf{1 6 - 1 8}}$ may stabilize higher oxidation state intermediates so they can be studied in more detail. Dianionic renditions of these ligands should allow for the synthesis of neutral higher oxidation state compounds; the transposition of carbon for boron at the linking positions of the $\mathrm{PY}_{5}$ or $\mathrm{Pz}_{4} \mathrm{Py}$ ligands is an established way to introduce the negative charges in the form of borates. ${ }^{19}$ Here, we introduce the new dianionic ligand $\mathrm{B}_{2} \mathrm{Pz}_{4} \mathrm{Py}$ and its use in the synthesis of iron imido complexes that are strong hydrogen atom acceptors.

\section{Results and discussion}

Details concerning the synthetic assembly of the $\mathrm{B}_{2} \mathrm{Pz}_{4} \mathrm{Py}$ ligand framework are given in Fig. 1. The most convenient ligand precursor, the $\left[\mathrm{B}_{2} \mathrm{Pz}_{4} \mathrm{LiPyH}\right]_{2}$ dimer shown in Fig. 1, can be prepared in two steps starting from 2,6-bis(trimethylstannyl) pyridine ${ }^{20}$ and bromo-(dimethylamino)phenyl borane. ${ }^{21}$ Mixing these two reagents together in toluene and stirring at room temperature for 24 hours yields a pale yellow semi solid upon removal of volatiles. This 2,6-diborylpyridyl species is stabilized by the strongly $\pi$ donating $\mathrm{NMe}_{2}$ groups, but does exhibit strong sensitivity to even weak donors and so is used without further purification in a reaction with a 3:1 mixture of freshly sublimed pyrazole and lithium pyrazolate. This leads to elimination of $\mathrm{HNMe}_{2}$ and production of gram quantities of $\left[\mathrm{B}_{2} \mathrm{Pz}_{4}\right.$ $\mathrm{LiPyH}]_{2}$, isolated in up to $81 \%$ yield as an air and water stable white solid. Crystals were obtained from THF/hexanes and the structure solution shows that the pyrazolyl nitrogens from opposing units coordinate a lithium counteranion in an approximately tetrahedral environment. The pyridine nitrogens are protonated to balance the second borate negative charge 


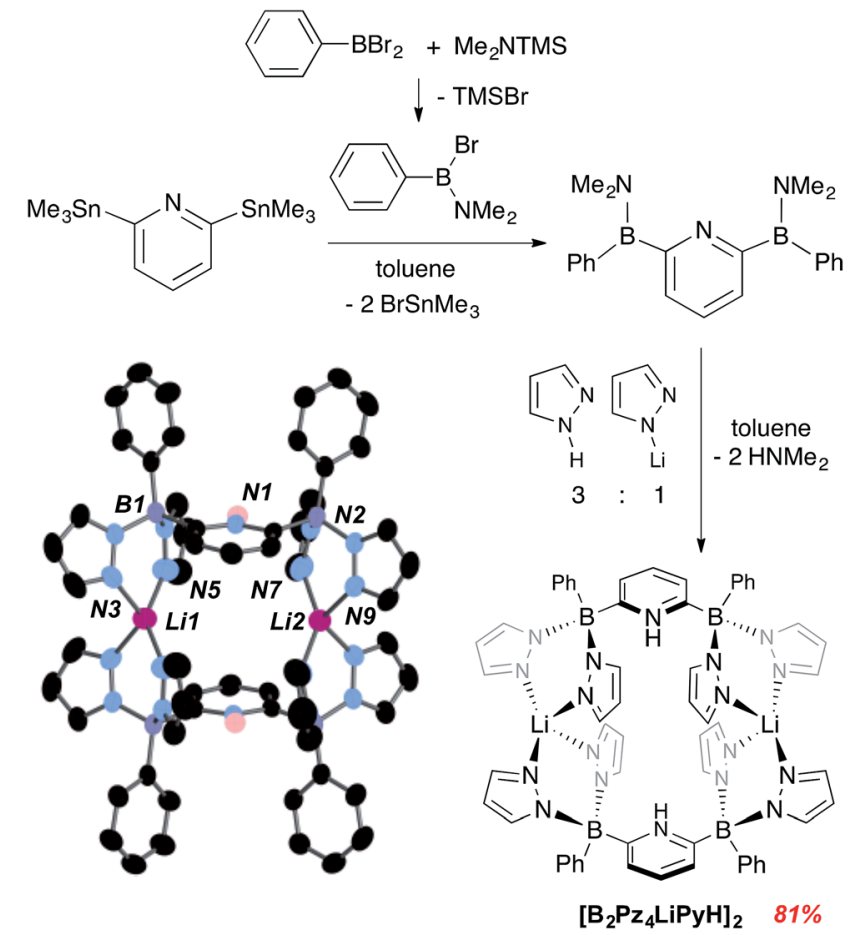

Fig. 1 Synthesis and structure of dianionic pentadentate ligand precursor $\left[\mathrm{B}_{2} \mathrm{Pz}_{4} \mathrm{LiPyH}\right]_{2}$. Thermal ellipsoids are drawn to the $50 \%$ probability level.

(Fig. 1). Metrical data within the structure are unremarkable and details can be found in the ESI (Fig. S1 $\dagger$ ).

For attachment to iron, this ligand precursor was taken up in THF and deprotonated using $\mathrm{LiO}^{\mathrm{i}} \mathrm{Pr}$; this solution of in situ generated dilithio salt was then transferred to a suspension of $\mathrm{FeBr}_{2}$ in THF (Scheme 1) to form the Fe(II) complex 1·THF. Yellow-green crystals of this air and moisture sensitive, paramagnetic compound were obtained, and its structure confirmed via X-ray crystallography (Fig. S2 $\dagger$ ). The ligand coordinates in a square-pyramidal array, while the THF ligand occupies the position trans to the pyridyl group to form an overall octahedral geometry. An Evan's method measurement yields a $\mu_{\text {eff }}$ of 5.64 B. M., consistent with a high spin (HS) electron configuration with 4 unpaired electrons $(S=2)$. The $80 \mathrm{~K}$ Mossbauer spectrum of 1 . THF features a quadrupole doublet with parameters of $\delta=$ $1.13 \mathrm{~mm} \mathrm{~s}^{-1}$ and $\Delta E_{\mathrm{Q}}=2.40 \mathrm{~mm} \mathrm{~s}^{-1}$, consistent with an $S=2$ iron(II) species (Fig. S3† and Table 1). ${ }^{22-25}$ DFT computations using the B3PW91 functional indicate that the HS $S=2$ configuration is $10.2 \mathrm{kcal} \mathrm{mol}^{-1}$ lower in energy than the low spin (LS) $S=0$ configuration (Table S1 $\dagger$ ).

Low valent iron complexes can react with nitrene transfer agents to generate higher oxidation state imido derivatives. ${ }^{\mathbf{2 6 - 4 1}}$ Complex 1-THF was thus reacted with organic azides, $\mathrm{RN}_{3}$, of varying electronic properties $\left(\mathrm{R}=\right.$ adamantyl, $\mathrm{C}_{6} \mathrm{H}_{4}-p-{ }^{t} \mathrm{Bu}$ and $\left.\mathrm{SO}_{2} \mathrm{C}_{6} \mathrm{H}_{4}-p{ }^{t} \mathrm{Bu}\right)$ using dry benzene as a solvent in an attempt to generate isolable, neutral $\mathrm{Fe}(\mathrm{Iv})$ imido derivatives. In each case, the product was the corresponding Fe(III) amido complex $\mathbf{2} \cdot \mathbf{R}$ (Scheme 1), isolated in moderate yields. Amidos $\mathbf{2} \cdot \mathbf{R}$ were all fully characterized, including via X-ray crystallography for $\mathbf{2} \cdot \mathbf{A r}$ (shown in Fig. 2A) and 2.Ad (Fig. S5 $\dagger$ ). All are paramagnetic with
$\left[\mathrm{B}_{2} \mathrm{Pz}_{4} \mathrm{LiPyH}\right]_{2}$

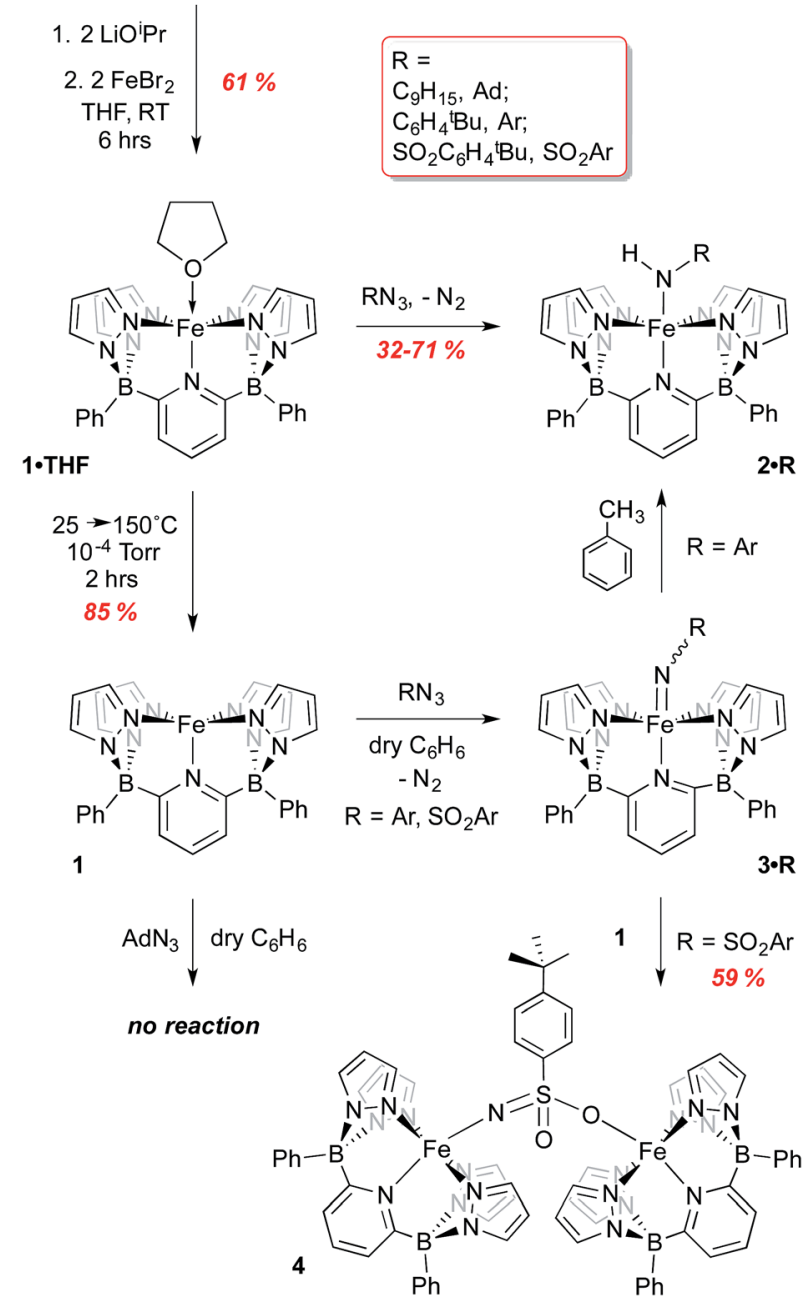

Scheme 1 Synthesis of iron(॥) complexes of $\left[\mathrm{B}_{2} \mathrm{Pz}_{4} \mathrm{LiPyH}\right]_{2}$ and their reactions with organoazides $\mathrm{RN}_{3}\left(\mathrm{R}=\right.$ adamantyl, $\mathrm{C}_{6} \mathrm{H}_{4}{ }^{t} \mathrm{Bu}$, $\mathrm{SO}_{2} \mathrm{C}_{6} \mathrm{H}_{4}{ }^{t} \mathrm{Bu}$ ).

Table 1 Experimental and calculated Mössbauer parameters for compounds $1 \cdot \mathrm{THF}, 2 \cdot \mathrm{Ar}$, and $3 \cdot \mathrm{Ar}$

\begin{tabular}{llllll}
\hline & \multicolumn{2}{l}{$\begin{array}{l}\text { Mössbauer parameters } \\
\text { (experimental) }\end{array}$} & & \multicolumn{2}{l}{$\begin{array}{l}\text { Mössbauer parameters } \\
\text { (calculated) }\end{array}$} \\
\cline { 2 - 3 } $\mathrm{Cmpd}$ & $\delta\left(\mathrm{mm} \mathrm{s}^{-1}\right)$ & $\Delta E_{\mathrm{Q}}\left(\mathrm{mm} \mathrm{s}^{-1}\right)$ & & $\delta\left(\mathrm{mm} \mathrm{s}^{-1}\right)$ & $\Delta E_{\mathrm{Q}}\left(\mathrm{mm} \mathrm{s}^{-1}\right)$ \\
\hline $\mathbf{1} \cdot \mathbf{T H F}$ & 1.13 & 2.40 & 0.91 & 2.94 \\
$\mathbf{2} \cdot \mathbf{A r}$ & 0.26 & 1.24 & 0.24 & 1.00 \\
$3 \cdot \mathbf{A r}$ & 0.20 & 1.96 & 0.31 & 1.79
\end{tabular}

${ }^{a} \mathbf{1} \cdot \mathbf{T H F}$ and $2 \cdot$ Ar data collected at $80 \mathrm{~K}, 3 \cdot$ Ar data collected at $5 \mathrm{~K}$.

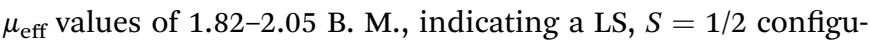
ration, and exhibit sharp $\mathrm{N}-\mathrm{H}$ stretches in the IR spectrum at $3262(\mathbf{2} \cdot \mathbf{A d}), 3303(\mathbf{2} \cdot \mathbf{A r})$ and $3071\left(\mathbf{2} \cdot \mathbf{S O}_{2} \mathbf{A r}\right) \mathrm{cm}^{-1}$. EPR spectroscopy of $2 \cdot$ Ar further confirms that it is an $S=1 / 2$ species (see ESI, Fig. S6 $\dagger$ ). In the UV-vis spectra, these amides exhibit low energy absorptions at $625 \mathrm{~nm}(\mathbf{2} \cdot \mathbf{A d})$ and $829 \mathrm{~nm}(\mathbf{2} \cdot \mathbf{A r})$. 


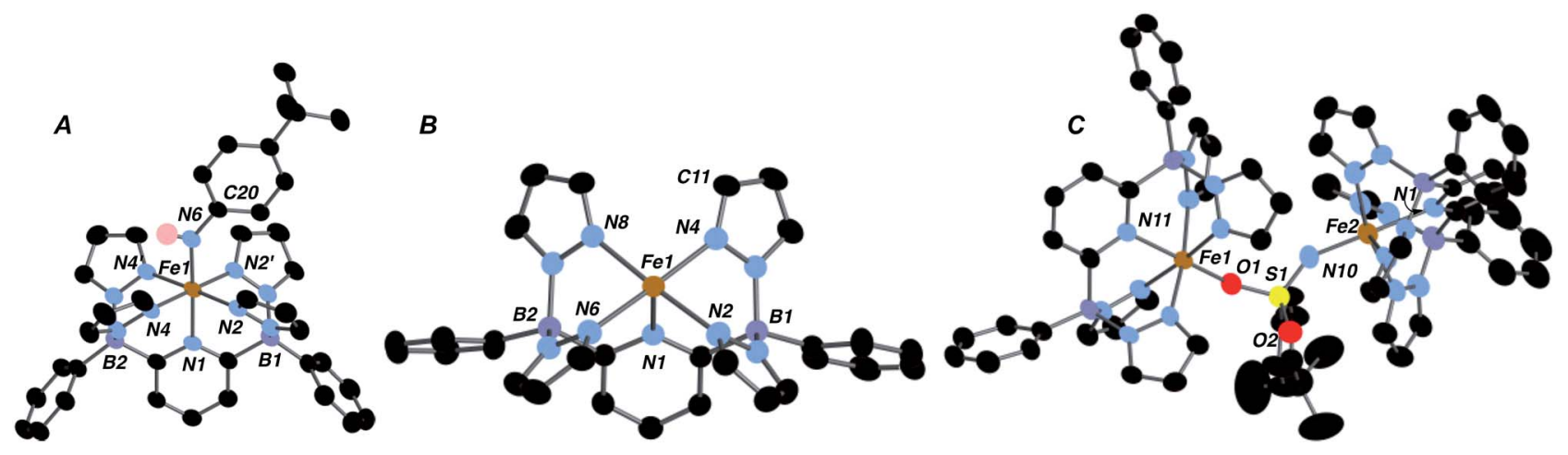

Fig. 2 Molecular structures (50\% ellipsoids) of compounds $2 \cdot \operatorname{Ar}(A), 1$ (B) and 4 (C). Selected metrical parameters (distances in $\AA$, angles in ${ }^{\circ}$ ) for 2.Ar: $\mathrm{Fe}(1)-\mathrm{N}(1), 1.994(3) ; \mathrm{Fe}(1)-\mathrm{N}(2), 1.982(2) ; \mathrm{Fe}(1)-\mathrm{N}(4), 2.002(2) ; \mathrm{Fe}(1)-\mathrm{N}(6), 1.869(3) ; \mathrm{N}(2)-\mathrm{Fe}(1)-\mathrm{N}(6), 91.97(11) ; \mathrm{N}(4)-\mathrm{Fe}(1)-\mathrm{N}(6), 89.45(11)$; $\mathrm{N}(1)-\mathrm{Fe}(1)-\mathrm{N}(6), 178.34(16) ; \mathrm{Fe}(1)-\mathrm{N}(6)-\mathrm{C}(20), 139.0(3)$. The aryl amido ligand plane lies along the plane bisecting the $\mathrm{N}(2)-\mathrm{Fe}(1)-\mathrm{N}\left(2^{\prime}\right)$ and $\mathrm{N}(4)-$ $\mathrm{Fe}(1)-\mathrm{N}\left(4^{\prime}\right)$ angles. Selected metrical parameters for 1: Fe(1)-N(1), 2.098(2); Fe(1)-N(2), 2.159(2); Fe(1)-N(4), 2.101(2); Fe(1)-N(6), 2.130(2); Fe(1)$\mathrm{N}(8), 2.089(2)$. Selected metrical parameters for 4: $\mathrm{Fe}(1)-\mathrm{O}(2), 1.913(3) ; \mathrm{Fe}(1)-\mathrm{N}(11), 2.111(4) ; \mathrm{S}(1)-\mathrm{O}(1), 1.460(3) ; \mathrm{S}(1)-\mathrm{O}(2), 1.513(3) ; \mathrm{S}(1)-\mathrm{N}(10)$, 1.503(4); $\mathrm{Fe}(2)-\mathrm{N}(10), 1.909(4) ; \mathrm{Fe}(2)-\mathrm{N}(1), 2.144(4) ; \mathrm{Fe}(1)-\mathrm{O}(2)-\mathrm{S}(1), 151.0(2) ; \mathrm{Fe}(2)-\mathrm{N}(10)-\mathrm{S}(1), 148.6(3)$.

Qualitatively, the rate of the reaction was related to the nature of $\mathrm{R}$; for adamantyl azide, the reaction required heating, while for the aryl and sulfonyl azides, gas evolution was immediate upon addition of the azide to solutions of $\mathbf{1}$-THF. For the aryl azide, an initially formed brown solution turned green over a couple of hours, suggesting the intermediacy of a transient species. We surmised that the path to amido products $2 \cdot \mathbf{R}$ may involve the predicted $\mathrm{Fe}(\mathrm{Iv})$ imido species, which then abstract a hydrogen atom from the liberated THF ligand to form the observed products; indeed, when THF- $d_{8}$ is added, an N-D stretch in the IR spectrum at $2451 \mathrm{~cm}^{-1}$ for $\mathbf{2} \cdot \mathbf{A r}-d_{1}$ is observed, but full conversion to $\mathbf{2} \cdot \mathbf{A r}$ takes significantly longer and the sample is only partially deuterated (see below). The products of $\mathrm{H}$ atom abstraction from THF in this reaction were not definitively identified, but THF is the most likely source of hydrogen atoms in this system.

We therefore hypothesized that removal of hydrogen atom donors from the system would be necessary in order to observe the putative $\mathrm{Fe}(\mathrm{rv})$ imido compounds. Heating solid samples of isolated $1 \cdot \mathbf{T H F}$ to $150{ }^{\circ} \mathrm{C}$ under high vacuum for a few hours resulted in removal of the ligated THF, forming THF-free $\mathbf{1}$ in excellent yield. Recrystallization from very dry benzene/pentane mixtures allowed for determination of its molecular structure, which is shown in Fig. 2B. The geometry at iron is square pyramidal, and the complex retains the HS, $S=2$, configuration found in 1.THF, with a $\mu_{\text {eff }}$ of 5.33 B. M. DFT computations reproduce the experimental structure accurately and indicate the HS configuration is $14.4 \mathrm{kcal} \mathrm{mol}^{-1}$ more stable than the LS configuration in this 5 coordinate derivative (Table S2 $\dagger$ ). The "naked" 5-coordinate complex is somewhat unusual, but it should be noted that in the crystal structure, the complex packs in dimeric units in which $\mathrm{C}(11)$ comes into close contact with the iron center of the adjacent molecule $(\mathrm{Fe}(1) \cdots \mathrm{C}(11)=3.282$ $\AA$ A Fig. S7†). In solution, however, this vacant coordination site is available for reaction with Lewis bases and other reagents.

With THF-free 1 in hand, we examined its reactions with the three azides $\mathrm{RN}_{3}$ in hydrogen atom transfer (HAT) inert solvents such as benzene or bromobenzene (refer back to Scheme 1). It was necessary to rigorously exclude polar solvents and impurities to avoid producing amides 2; indeed, even working up the reactions in hexanes, which likely contain tertiary $\mathrm{C}-\mathrm{H}$ bonds, led to the amido products and thus it was necessary to employ pure, dry pentane or $\mathrm{Me}_{3} \mathrm{SiOSiMe}_{3}$ to prevent formation of the amido complexes. Again, the facility of the reaction between 1 and the azides was dependent on the nature of the azide $\mathrm{R}$ substituent. Surprisingly, for adamantyl azide, no reaction was observed with 1, even upon heating to $60^{\circ} \mathrm{C}$ in bromobenzene. Indeed, there was no visual or spectroscopic (UV-vis) evidence for interaction of the $\mathrm{AdN}_{3}$ reagent with 1. Only when a species capable of contributing a hydrogen atom (THF or toluene) was introduced did the reaction proceed, then giving $\mathbf{2}$-Ad directly. This observation suggests that an Fe imido complex is not readily formed for this azide and that reaction of $\mathbf{1}$ with this bulky azide requires the aid of a hydrogen atom donor in order to proceed.

In contrast, reaction with the sulfonyl azide $\mathrm{N}_{3} \mathrm{SO}_{2} \mathrm{Ar}$ was rapid at room temperature, evidenced by vigorous gas evolution upon dropwise addition of the azide to solutions of 1 . The product isolated and characterized by X-ray crystallography was the dinuclear $\mathrm{Fe}(\mathrm{III})-\mathrm{Fe}$ (III) species 4, the molecular structure of which is shown in Fig. 2C, along with selected metrical data. The $\mathrm{Fe}(1)-\mathrm{O}(2)$ and $\mathrm{Fe}(2)-\mathrm{N}(10)$ distances are consistent with $\mathrm{Fe}(\mathrm{III})$-element single bonds, while the $\mathrm{S}(1)-\mathrm{N}(10)$ distance of 1.503(4) $\AA$ is indicative of some multiple bonding in this linkage. Compound 4 likely forms through the rapid formation of an $\mathrm{Fe}(\mathrm{Iv})$ imido, $\mathbf{3} \cdot \mathbf{S O}_{2} \mathbf{A r}$, which rapidly picks up a second equivalent of $\mathrm{Fe}(\mathrm{II}) \mathbf{1}$ to give the observed dinuclear species (Scheme 1). ${ }^{42}$ Attempts to observe $\mathbf{3} \cdot \mathbf{S O}_{2} \mathbf{A r}$ by using an "inverse addition" strategy, or by treating $\mathbf{4}$ with an excess of azide reagent met with failure and only 4 was produced. When isolated samples of 4 were treated with an excess of THF at room temperature, a $1: 1$ mixture of $2 \cdot \mathbf{S O}_{2} \mathbf{A r}$ and $\mathbf{1} \cdot \mathbf{T H F}$ was produced rapidly.

However, when the aryl azide ${ }^{t} \mathrm{BuC}_{6} \mathrm{H}_{4} \mathrm{~N}_{3}$ was employed in reaction with $\mathbf{1}$, evidence for formation of $\mathbf{3} \cdot \mathbf{A r}$ was observed. Rapid evolution of $\mathrm{N}_{2}$ was accompanied by a deepening of the 
light green color of $\mathbf{1}$ to a brown suspension from which dark green, solid 3.Ar could be isolated in $67 \%$ yield. The product is paramagnetic with a $\mu_{\text {eff }}$ value of 2.87 B. M., indicative of an $S=$ 1 spin state, as determined via the Evans method on freshly prepared samples. Unfortunately, despite several attempts, crystals of this crucial material could not be obtained, likely due to its propensity to convert to amido complex $2 \cdot \mathbf{A r}$. Consequently, 3·Ar was examined through DFT studies and the computed structure was obtained.

The computed structures of the LS $(S=0)$, HS $(S=2)$ and $S=$ 1 spin states of $\mathbf{3} \cdot \mathbf{A r}$ indicate that the last is the most stable by $12.1 \mathrm{kcal} \mathrm{mol}^{-1}$ over the LS configuration (Tables S3 and S4 $\dagger$ ). The structure is similar in geometry to that of $\mathrm{Fe}(\mathrm{III})$ amido complex $\mathbf{2} \cdot \mathbf{A r}$ (Fig. 3), but exhibits a much shorter Fe-N distance of $1.696 \AA$, in line with other Fe imido complexes, ${ }^{26,43,44}$ and a wider $\mathrm{Fe}-\mathrm{N}-\mathrm{C}_{\text {ipso }}$ angle of $143.33^{\circ}$. The $\mathrm{N}-\mathrm{C}_{\mathrm{ipso}}$ distance of $1.349 \AA$, coupled with alternation of long-short-long bond distances within the aryl ring suggest the NAr ligand is best described an imido based radical $;^{37}$ indeed, the highest energy SOMO orbital shows significant localization on the imido nitrogen (Fig. 3, inset) and the calculated spin density population (Fig. S8†) is also in agreement with this description.

Mössbauer spectroscopy of 3 $\cdot$ Ar was performed to evaluate the oxidation state of this species. While the Mössbauer spectrum of $\mathbf{3} \cdot \mathbf{A r}$ at $80 \mathrm{~K}$ is broadened, the spectrum at $5 \mathrm{~K}$ (Fig. $4 \mathrm{~B}$ ) is well-resolved. It contains a major quadrupole doublet (red component, $80 \%$ of all iron) with parameters of $\delta=0.20 \mathrm{~mm} \mathrm{~s}^{-1}$ and $\Delta E_{\mathrm{Q}}=1.96 \mathrm{~mm} \mathrm{~s}^{-1}$ as well as two additional iron species (see Fig. 4 caption), including one that is consistent with the presence of $\mathbf{2} \cdot \mathbf{A r}$, likely formed from $\mathbf{3} \cdot \mathbf{A r}$ during transport and handling of the sample. Spin-quantitated EPR analysis further supports this minor component as being an $S=1 / 2$ species (see ESI $\dagger$ ). Importantly, the isomer shift of the major species corresponding to $\mathbf{3} \cdot \mathbf{A r}$ is similar to that for $\mathbf{2} \cdot \mathbf{A r}$ (an iron(III) species), suggestive of similar oxidation states of iron in both complexes. In addition, previously reported iron(Iv) imido species typically have low isomer shifts: the iron(Iv)-tosylamido species of Que

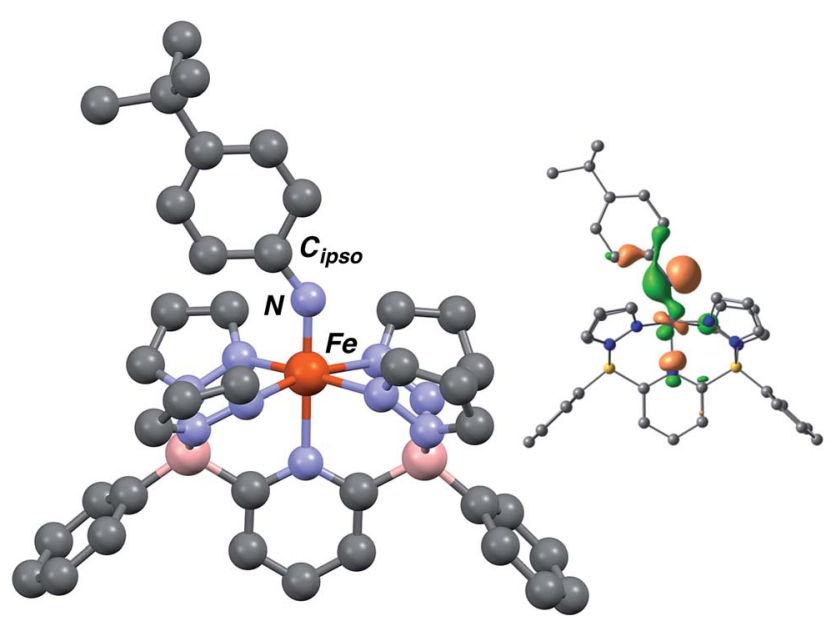

Fig. 3 Computed structure of $3 \cdot$ Ar at the B3PW91 level of theory Selected bond distances and angles $(\AA)$ : $\mathrm{Fe}-\mathrm{N}, 1.696 \AA \AA \mathrm{A} ; \mathrm{Fe}-\mathrm{N}-\mathrm{C}_{\text {ipso, }}$ $143.33^{\circ}$. Depiction of the highest energy SOMO orbital (upper right).

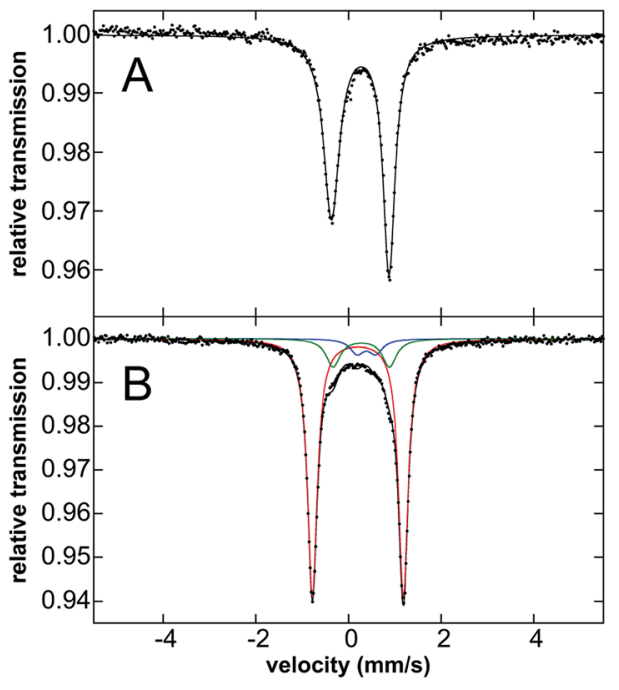

Fig. $480 \mathrm{~K}$ Mössbauer spectra of solid (A) 2. Ar collected at $80 \mathrm{~K}$ and (B) $3 \cdot$ Ar collected at $5 \mathrm{~K}$. The two minor impurities in the $3 \cdot$ Ar sample have Mossbauer parameters of $\delta=0.26 \mathrm{~mm} \mathrm{~s}^{-1}, \Delta E_{\mathrm{Q}}=1.24 \mathrm{~mm} \mathrm{~s}^{-1}$ (green, $13 \%$ of total iron) and $\delta=0.39 \mathrm{~mm} \mathrm{~s}^{-1}, \Delta E_{\mathrm{Q}}=0.40 \mathrm{~mm} \mathrm{~s}^{-1}$ (blue, $7 \%$ of total iron).

and co-workers ${ }^{32}\left(\delta=0.02 \mathrm{~mm} \mathrm{~s}^{-1}\right)$, the iron(Iv)-imido corrolazine complex of Goldberg et al. ${ }^{45}\left(\delta=-0.05 \mathrm{~mm} \mathrm{~s}^{-1}\right)$, the iron(Iv) pincer imido reported recently by Mindiola et al. $(\delta=$ $\left.-0.09 \mathrm{~mm} \mathrm{~s}^{-1}\right)^{\mathbf{4 6}}$ and the novel carbene-stabilized bis-imido iron(Iv) complexes of Deng $\left(\delta=-0.29 \text { to }-0.52 \mathrm{~mm} \mathrm{~s}^{-1}\right)^{47}$ are pertinent examples. Thus, the Mössbauer data is most consistent with an iron(III) description for $\mathbf{3} \cdot \mathbf{A r}$. This assignment was further corroborated by computing the Mössbauer data using methodology developed by Neese et al. $;^{\mathbf{4 8}}$ as can be seen in Table 1 , the computed values are in broad agreement with the experimental values for both $\mathbf{2} \cdot \mathbf{A r}$ and $\mathbf{3} \cdot \mathbf{A r}$, taking into account the limitations of such calculations.

Taken together, the above data suggests the nature of $\mathbf{3} \cdot \mathbf{A r}$ is best described as a LS Fe(III) $\left(\mathrm{d}^{5}, S=1 / 2\right)$ iron center bonded to an $S=1 / 2$ imido radical species. Such imido radical formulations have been proposed in other systems, both for iron $^{37}$ and cobalt. $^{49-52}$ In $\mathbf{3} \cdot \mathbf{A r}$, the two spins reside in orthogonal orbitals and so an $S=1$ system is the result. This description also accounts for why this compound so readily converts to $\mathbf{2} \cdot \mathbf{A r}$ in the presence of even weak hydrogen atom donors, such as toluene (the Bond Dissociation Energy, BDE, for the benzylic C-H bond of toluene is $\left.\approx 89 \mathrm{kcal} \mathrm{mol}^{-1}\right) .{ }^{53}$ While the lower energy SOMO is essentially metal-based (see Fig. S8, ESI $\dagger$ ), the orbital associated with the HAT reactivity is imido nitrogen-based and optimally oriented for accepting a hydrogen atom donor (Fig. 3, inset). Experiments are also consistent with this picture. Quantitative analysis of the rate of the reaction of $\mathbf{3} \cdot \mathbf{A r}$ with varying amounts of toluene (20-300 equivalents) was conveniently done by following the reactions by UV-vis spectroscopy at room temperature (Fig. S9, ESI $\dagger$ ). The process is shown to be first order in [toluene], suggesting a bimolecular HAT reaction involving the benzylic toluene hydrogens as the source of the amido hydrogen in $2 \cdot$ Ar. From the plot of $k_{\text {obs }} v s$. [toluene] (Fig. S10 and S11, ESI $\dagger$ ), a second order rate constant of $1.60(5) \times 10^{-3} \mathrm{M}^{-1} \mathrm{~s}^{-1}$ is 
obtained. This is comparable to that of $9.1 \pm 0.9 \times 10^{-3} \mathrm{M}^{-1} \mathrm{~s}^{-1}$ observed in the bimolecular HAT reaction between a 4 coordinate beta-diketiminato supported Fe(III) imido and 1,4-cyclohexadiene at $-51{ }^{\circ} \mathrm{C} .{ }^{36}$ While comparison of these two systems is tempered by the different conditions under which the rates were measured, we note that the lower coordinate iron imido is unreactive towards toluene, indicating qualitatively that the present system is highly reactive towards even weak hydrogen atom donors in comparison. Indeed, a transition state for HAT from toluene to 3.Ar was located and exhibits a near linear $\mathrm{N}-\mathrm{H}-\mathrm{C}$ vector with a low barrier of $6.4 \mathrm{kcal} \mathrm{mol}^{-1}$ for hydrogen transfer (Fig. S12 and Table S5 $\dagger$ ). The computed homolytic BDE for the $\mathrm{N}-\mathrm{H}$ bond in $2 \cdot$ Ar was found to be $91.3 \mathrm{kcal} \mathrm{mol}^{-1}$, slightly greater than values found in Holland's beta-diketiminato amido complexes, ${ }^{36}$ and contributes to the exothermicity of HAT to the imido radical ligand in $\mathbf{3} \cdot \mathbf{A r}$.

Since the product of HAT $\mathbf{2} \cdot \mathbf{A r}$ is paramagnetic, quantification of the $\mathrm{NH} v s$. ND isotopologues was challenging; furthermore, we found by IR spectroscopy that the major isotopologue produced from reaction of $\mathbf{3} \cdot \mathbf{A r}$ with deuterated THF or toluene was in fact the $\mathrm{NH}$ isotopologue, suggesting that even the small amount of residual protons in the deuterated solvent are able to compete in these HAT reactions. Nonetheless, by integrating the $\mathrm{N}-\mathrm{H}$ vs. the $\mathrm{N}-\mathrm{D}$ stretching bands in the IR spectrum of a sample of $\mathbf{2} \cdot \mathbf{A r}-d_{n}(n=0,1)$ generated by gentle heating of $\mathbf{3} \cdot \mathbf{A r}$ in THF- $d_{8}\left(0.23 \%\right.$ THF- $\left.d_{7}\right)$, a $k_{\mathrm{H}} / k_{\mathrm{D}}$ of $567 \pm 16$ was estimated. This experiment was repeated three times and a similar result was observed when toluene- $d_{8}$ was employed. The calculated intensities of the $\mathrm{N}-\mathrm{H}$ vs. $\mathrm{N}-\mathrm{D}$ stretching frequencies indicate that they are of a similar magnitude, with the $\mathrm{N}-\mathrm{D}$ stretch being less intense by a factor of 1.8 ; even with this correction a $k_{\mathrm{H}} / k_{\mathrm{D}}$ of $\approx 315$ is obtained. While certainly unusual, we note that KIEs of this magnitude are a hallmark of the HAT reaction. For example Holland et al. report a value of $105 \pm 28$ in the HAT from cyclohexadiene to a beta-diketiminato ligand supported Fe(III) imido complex. ${ }^{36}$ This value was obtained by taking the ratio of measured rate constants obtained using undeuterated and fully deuterated cyclohexadiene substrates and does not account for the possibility of competitive $\mathrm{H}$ atom transfer from residual protons in the deuterated substrate; it therefore may represent a low estimate of the true $k_{\mathrm{H}} / k_{\mathrm{D}}$ in this system. Using a more accurate method, Meyer et al. report a "colossal" $k_{\mathrm{H}} / k_{\mathrm{D}}$ of $439 \pm 8$ in the transfer of a hydrogen atom from $\mathrm{N}$ to a substrate in an osmium amide/imide system. ${ }^{54}$ Thus, such reactions do feature very large KIE, and while the value obtained for the conversion of $\mathbf{3} \cdot \mathbf{A r}$ to $\mathbf{2} \cdot \mathbf{A r}$ here likely has a large error associated with it, the key point is that it is much larger than a conventional KIE. We calculate a $k_{\mathrm{H}} / k_{\mathrm{D}}$ of 5.82 by substituting $\mathrm{D}$ for $\mathrm{H}$ in the transition state calculation; clearly, the experimental value is much greater than that and supports a HAT mechanism with a substantial tunneling component during the transfer of the hydrogen atom.

Unlike Betley's imido radical system, ${ }^{37,40}$ which also abstracts hydrogen atoms from suitable donors, we do not observe significant $\mathrm{N}-\mathrm{C}$ bond formation via a radical-rebound process. Thus, our system does not mediate $\mathrm{C}-\mathrm{H}$ bond amination, and we detect less that $4 \%$ of the $\operatorname{Ar}\left(\mathrm{PhCH}_{2}\right) \mathrm{NH}$ amine product that would be expected if this were occurring in the reaction of $\mathbf{3} \cdot \mathbf{A r}$ with toluene. Following the intrinsic reaction coordinates from the TS for hydrogen atom transfer (Fig. S12 $\dagger$ ) leads to the full dissociation of the benzyl radical and there is no clear driving force for any rebound of the formed radical. Every attempt to rebound the radical leads back to the $\mathrm{C}-\mathrm{H}$ activation $\mathrm{TS}$ so that it seems that there is no energetically accessible TS leading to $\mathrm{C}-\mathrm{N}$ bond formation. We speculate that the change from a LS configuration in $\mathbf{2} \cdot \mathbf{A r}$ to a HS configuration in the Fe(II) amine product may play a role in the low level of $\mathrm{C}-\mathrm{N}$ bond formation in this system.

\section{Conclusions}

In conclusion, we have generated and characterized a 6 coordinate $\mathrm{Fe}(\mathrm{III})$ imido radical via reaction of a 5 coordinate $\mathrm{Fe}$ (II) species with an aryl azide. It appears that the affinity of the imido group in $\mathbf{3} \cdot \mathbf{A r}$ for hydrogen atoms is quite high relative to previously reported, lower coordinate systems, ${ }^{36,37}$ an observation which may be related to the higher coordination number and anionic nature of the ligand in $\mathbf{3} \cdot \mathbf{A r}$, which pushes significant spin density onto the imido nitrogen. In combination with the sterically modest (i.e., no ortho groups on the aryl substituent) and bent para- $^{t} \mathrm{Bu} \mathrm{N}$-aryl group, this effect results in an imido radical ligand that exhibits powerful hydrogen atom acceptor ability.

\section{Acknowledgements}

Funding for this work was provided by NSERC of Canada (Discovery Grant) the Canada Research Chair secretariat (Tier I CRC 2013-2020) to W. E. P., the National Science Foundation (CHE-1454370) to M. L. N. and by an Alfred P. Sloan Research Fellowship to M. L. N. The Alexander von Humboldt Foundation (W. E. P. and L. M.) and the Chinese academy of Science (L. M.) are acknowledged for financial support. L. M. is member of the Institut Universitaire de France. L. M. and W. P. also acknowledge CNRS for a bilateral grant through the PICS program. CalMip is acknowledeged for a generous grant of computing time. D. M. S. thanks the University of Calgary for an Eyes High Postdoctoral Fellowship. Prof. Hans-Jörg Himmel of the Ruprecht-Karls-Universität Heidelberg is acknowledged for helpful discussions.

\section{Notes and references}

1 A. Grohmann, Dalton Trans., 2010, 39, 1432-1440.

2 R. T. Jonas and T. D. P. Stack, J. Am. Chem. Soc., 1997, 119, 8566-8567.

3 M. E. de Vries, M. R. La Crois, G. Roelfes, H. Kooijman, A. L. Spek, R. L. Hage and B. Feringa, Chem. Commun., 1997, 1549-1550.

4 R. J. M. Klein Gebbink, R. T. Jonas, C. R. Goldsmith and T. D. P. Stack, Inorg. Chem., 2002, 41, 4633-4641.

5 C. R. Goldsmith, R. T. Jonas, A. P. Cole and T. D. P. Stack, Inorg. Chem., 2002, 41, 4642-4652. 
6 D. Z. Zee, T. Chantarojsiri, J. R. Long and C. J. Chang, Acc. Chem. Res., 2015, 48, 2027-2036.

7 J. P. Bigi, T. E. Hanna, W. H. Harman, A. Chang and C. J. Chang, Chem. Commun., 2010, 46, 958-960.

8 Y. Sun, J. P. Bigi, N. A. Piro, M. L. Tang, J. R. Long and C. J. Chang, J. Am. Chem. Soc., 2011, 133, 9212-9215.

9 Y. Sun, J. Sun, J. R. Long, P. Yang and C. J. Chang, Chem. Sci., 2013, 4, 118-124.

10 H. I. Karunadasa, C. J. Chang and J. R. Long, Nature, 2010, 464, 1329-1333.

11 D. J. Wasylenko, C. Ganesamoorthy, J. Borau-Garcia and C. P. Berlinguette, Chem. Commun., 2011, 47, 4249-4251.

12 D. J. Wasylenko, R. D. Palmer, E. Schott and C. P. Berlinguette, Chem. Commun., 2012, 48, 2107-2109.

13 D. W. Crandell, S. Ghosh, C. P. Berlinguette and M.-H. Baik, ChemSusChem, 2015, 8, 844-852.

14 V. S. Thoi, Y. Sun, J. R. Long and C. J. Chang, Chem. Soc. Rev., 2013, 42, 2388-2400.

15 T. J. Morin, B. Bennett, S. V. Lindeman and J. R. Gardinier, Inorg. Chem., 2008, 47, 7468-7470.

16 L. Tong, Y. Wang, L. Duan, Y. Xu, X. Cheng, A. Fischer, M. S. G. Ahlquist and L. Sun, Inorg. Chem., 2012, 51, 33883398.

17 K. J. Young, M. K. Takase and G. W. Brudvig, Inorg. Chem., 2013, 52, 7615-7622.

18 I. Siewert and J. Gałęzowska, Chem.-Eur. J., 2015, 21, 27802784.

19 S. Trofimenko, Scorpionates: The Coordination Chemistry of Polypyrazolylborate Ligands, Imperial College Press, London, 1999.

20 U. S. Schubert and C. Eschbaumer, Org. Lett., 1999, 1, 10271029.

21 P. A. Barfield, M. F. Lappert and J. Lee, J. Chem. Soc. A, 1968, 554-559.

22 P. Gütlich, E. Bill and A. E. Trautwein, Mössbauer Spectroscopy and Transition Metal Chemistry: Fundamentals and Applications, Springer-Verlag, Berlin, Heidelberg, 2011.

23 S. C. Bart, K. Chłopek, E. Bill, M. W. Bouwkamp, E. Lobkovsky, F. Neese, K. Wieghardt and P. J. Chirik, J. Am. Chem. Soc., 2006, 128, 13901-13912.

24 D. Benito-Garagorri, L. G. Alves, M. Puchberger, K. Mereiter, L. F. Veiros, M. J. Calhorda, M. D. Carvalho, L. P. Ferreira, M. Godinho and K. Kirchner, Organometallics, 2009, 28, 6902-6914.

25 L. R. Widger, Y. Jiang, M. A. Siegler, D. Kumar, R. Latifi, S. P. de Visser, G. N. L. Jameson and D. P. Goldberg, Inorg. Chem., 2013, 52, 10467-10480.

26 S. D. Brown, T. A. Betley and J. C. Peters, J. Am. Chem. Soc., 2003, 125, 322-323.

27 M. P. Jensen, M. P. Mehn and L. Que, Angew. Chem., Int. Ed., 2003, 42, 4357-4360.

28 S. D. Brown and J. C. Peters, J. Am. Chem. Soc., 2005, 127, 1913-1923.

29 R. L. Lucas, D. R. Powell and A. S. Borovik, J. Am. Chem. Soc., 2005, 127, 11596-11597.

30 S. C. Bart, E. Lobkovsky, E. Bill and P. J. Chirik, J. Am. Chem. Soc., 2006, 128, 5302-5303.
31 N. A. Eckert, S. Vaddadi, S. Stoian, R. J. Lachicotte, T. R. Cundari and P. L. Holland, Angew. Chem., Int. Ed., 2006, 45, 6868-6871.

32 E. J. Klinker, T. A. Jackson, M. P. Jensen, A. Stubna, G. Juhász, E. L. Bominaar, E. Münck and L. Que, Angew. Chem., Int. Ed., 2006, 45, 7394-7397.

33 C. M. Thomas, N. P. Mankad and J. C. Peters, J. Am. Chem. Soc., 2006, 128, 4956-4957.

34 I. Nieto, F. Ding, R. P. Bontchev, H. Wang and J. M. Smith, J. Am. Chem. Soc., 2008, 130, 2716-2717.

35 A. C. Bowman, C. Milsmann, E. Bill, Z. R. Turner, E. Lobkovsky, S. DeBeer, K. Wieghardt and P. J. Chirik, J. Am. Chem. Soc., 2011, 133, 17353-17369.

36 R. E. Cowley, N. A. Eckert, S. Vaddadi, T. M. Figg, T. R. Cundari and P. L. Holland, J. Am. Chem. Soc., 2011, 133, 9796-9811.

37 E. R. King, E. T. Hennessy and T. A. Betley, J. Am. Chem. Soc., 2011, 133, 4917-4923.

38 M.-E. Moret and J. C. Peters, Angew. Chem., Int. Ed., 2011, 50, 2063-2067.

39 R. E. Cowley and P. L. Holland, Inorg. Chem., 2012, 51, 83528361.

40 E. T. Hennessy, R. Y. Liu, D. A. Iovan, R. A. Duncan and T. A. Betley, Chem. Sci., 2014, 5, 1526-1532.

41 J. Xiao and L. Deng, Dalton Trans., 2013, 42, 5607-5610.

42 J. A. Bellow, M. Yousif, A. C. Cabelof, R. L. Lord and S. Groysman, Organometallics, 2015, 34, 2917-2923.

43 R. E. Cowley, N. J. DeYonker, N. A. Eckert, T. R. Cundari, S. DeBeer, E. Bill, X. Ottenwaelder, C. Flaschenriem and P. L. Holland, Inorg. Chem., 2010, 49, 6172-6187.

44 A. K. Verma, T. N. Nazif, C. Achim and S. C. Lee, J. Am. Chem. Soc., 2000, 122, 11013-11014.

45 P. Leeladee, G. N. L. Jameson, M. A. Siegler, D. Kumar, S. P. de Visser and D. P. Goldberg, Inorg. Chem., 2013, 52, 4668-4682.

46 K. Searles, S. Fortier, M. M. Khusniyarov, P. J. Carroll, J. Sutter, K. Meyer, D. J. Mindiola and K. G. Caulton, Angew. Chem., Int. Ed., 2014, 53, 14139-14143.

47 L. Wang, L. Hu, H. Zhang, H. Chen and L. Deng, J. Am. Chem. Soc., 2015, 137, 14196-14207.

48 M. Römelt, S. Ye and F. Neese, Inorg. Chem., 2009, 48, 784-785. 49 V. Lyaskovskyy, A. I. O. Suarez, H. Lu, H. Jiang, X. P. Zhang and B. de Bruin, J. Am. Chem. Soc., 2011, 133, 12264-12273.

50 A. I. Olivos Suarez, H. Jiang, X. P. Zhang and B. de Bruin, Dalton Trans., 2011, 40, 5697-5705.

51 A. I. O. Suarez, V. Lyaskovskyy, J. N. H. Reek, J. I. van der Vlugt and B. de Bruin, Angew. Chem., Int. Ed., 2013, 52, 12510-12529.

52 M. Goswami, V. Lyaskovskyy, S. R. Domingos, W. J. Buma, S. Woutersen, O. Troeppner, I. Ivanović-Burmazović, H. Lu, X. Cui, X. P. Zhang, E. J. Reijerse, S. DeBeer, M. M. van Schooneveld, F. F. Pfaff, K. Ray and B. de Bruin, J. Am. Chem. Soc., 2015, 137, 5468-5479.

53 Y.-R. Luo, Comprehensive Handbook of Chemical Bond Energies, CRC Press, Boca Raton, FL, 2007.

54 M. H. V. Huynh and T. J. Meyer, Proc. Natl. Acad. Sci. U. S. A., 2004, 101, 13138-13141. 